\title{
PERTINENCIA DE LA CONTABILIDAD AMBIENTAL PARA CONTRIBUIR A LA SUSTENTABILIDAD DE LA RIQUEZA AMBIENTAL
}

\author{
PERTINENCE OF ENVIRONMENTAL ACCOUNTING FOR CONTRIBUTE TO THE \\ SUSTAINABILITY OF ENVIRONMENTAL WEALTH
}

Olga Inés Ceballos Rincón Universidad del Quindío Armenia, Colombia ORCID: https://orcid.org/0000-0002-4082-6451 Correo electrónico: oiceballos@uniquindio.edu.co

Ciro Alfonso Serna Mendoza Universidad de Manizales Manizales, Colombia ORCID: https://orcid.org/0000-0003-3192-9771 Correo electrónico: redesomciro@hotmail.com

Eutimio Mejía Soto Universidad del Quindío Armenia, Colombia

ORCID: https://orcid.org/0000-0002-7162-9928 Correo electrónico: eutimiomejia@uniquindio.edu.co

[Recibido: 18/06/2020 Aceptado: 01/08/2020 Publicado: 31/08/2020]

\section{RESUMEN}

Objetivo: Analizar el conocimiento y aceptación que la comunidad académica contable tiene referente al desarrollo y estructura de la Biocontabilidad, entendida como disciplina emergente que propende por contribuir a la sustentabilidad de la riqueza ambiental. Método: La investigación es descriptiva e inductiva, por cuanto identifica a través de una muestra la postura de los expertos contables en referencia a la pertinencia de la Contabilidad Ambiental y la Biocontabilidad para representar la riqueza natural en función de su sustentabilidad. Resultados: La comunidad académica contable ha avanzado en la aceptación de la necesidad de desarrollar una estructura para esta disciplina que permita la preparación y presentación de información de la riqueza ambiental, a través de un modelo diferente al contable-financiero. Conclusiones: Un sistema contable ambiental debe tener criterios de reconocimiento, métodos de valoración, información por presentar y por revelar, propios de la dimensión natural para informar y rendir cuentas con respecto a su objeto de estudio.

Palabras clave: Biocontabilidad; medición; reconocimiento; revelación; sustentabilidad.

\begin{abstract}
Objective: To analyze the knowledge and acceptance that the accounting academic community has regarding the development and structure of biocontability, understood as the emerging discipline that tends to contribute to the sustainability of the environmental wealth. Method: The develop research is descriptive and inductive, so far as it identifies through a sample the position of accounting experts regarding the environmental accounting and biocontability pertinence to represent natural wealth in function to the sustainability of it. Results: The study allows to know that the accounting academic community Has advanced in accepting the need to develop a structure for this discipline that allows the preparation and presentation of information on environmental wealth, through a different model as the financial-accounting. Conclusions: it concludes that an environmental accounting system must have recognition criteria, assessments methods, information to present and revealing the own natural dimension to inform and yield accounts regarding to its study objective.
\end{abstract}

Keywords: Biocontability; measurement; recognition; revelation; sustainability. 


\section{INTRODUCCIÓN}

La investigación busca identificar el conocimiento y aceptación que la comunidad científica contable tiene referente al tema de la Biocontabilidad, entendida como disciplina emergente que propende contribuir a la sustentabilidad de la riqueza ambiental (Ceballos y Mejía, 2016; Mejía y Ceballos, 2016; Suárez et al., 2020).

La Biocontabilidad tiene en cuenta cuatro aspectos de un sistema contable, como son: los criterios de reconocimiento de los elementos, métodos de valoración de la riqueza, información a presentar e información a revelar en los estados biocontables; tópicos que esta disciplina emergente desarrolla para las representaciones no monetarias e integrales de los recursos naturales, como lo indica la regulación contable privada (International Accounting Financial Board, 2018) y pública (International Public Sector Accounting Standards Board, 2014).

El primer tópico referente a los criterios de reconocimiento de los elementos de los estados contables permitió que el encuestado confrontara los criterios tradicionales como son la probabilidad de que cualquier beneficio económico asociado con la partida llegue a, o salga de la entidad y que la partida tenga un costo o valor que pueda ser medido con fiabilidad, además de cumplir con la relevancia y la representación fiel (Marco Conceptual IFRS, 2018), frente a los que reconoce la Teoría Tridimensional de la Contabilidad, aplicados a la dimensión ambiental (Álvarez, 2019), los cuales son: la existencia y el control de organización sobre la riqueza.

El reconocimiento es la etapa inicial del ciclo contable, proceso en el cual se definen qué partidas se incluyen en los reportes contables y en qué momento se debe hacer; determinadas la cantidad, la fecha y la clasificación de los elementos de los estados contables, lo cual depende de los criterios de reconocimientos establecidos en el sistema. Estos criterios se complementan con el método de valoración, el concepto de capital, mantenimiento de capital, presentación y revelación de información (Cooper e Ijiri, 2005, p. 609; Grajales y Sánchez, 2004, p. 295; Fowler, 2008, p. 485 y Álvarez, 2006, p. 28).

El segundo tópico refiere a los métodos para la valoración de la riqueza ambiental. La contabilidad tradicional únicamente ha utilizado métodos cuantitativos monetarios para la incorporación de los elementos en los estados contables. La Biocontabilidad propone como criterios de valoración los métodos cualitativos, cuantitativos no monetarios y multicriterio (García, Rodríguez y Ruiz, 2016). El nuevo Marco Conceptual (International Accounting Financial Board, 2018) ratifica que "los elementos reconocidos en los estados financieros se cuantifican en términos monetarios" (párr. 6.1), considerando como bases de medición el costo histórico y el valor corriente (valor razonable, valor en uso y valor de cumplimiento y costo corriente).

El análisis multicriterio es una propuesta valorativa ampliamente incluyente, incorpora datos basados en preferencias humanas, como son los valores crematísticos y socioculturales, y las valoraciones físicas relacionadas con la medición energética, de materiales y biogeofísica como la memoria energética (Gómez y De Groott, 2007). La postura de los encuestados da apertura a la indagación en profundidad del impacto de este método en la valoración de la riqueza ambiental en función de su sustentabilidad.

Otros métodos de valoración de la riqueza ambiental en función de su sustentabilidad, alternos a los propuestos en el instrumento aplicado, que fueron expresados por algunos expertos son los relacionados con las ciencias puras, como la química, la física y la biología, que incluyen mediciones cuantitativas de volumen, masa y flujos físicos; además, los beneficios del entorno, las simulaciones y la creación de escenarios controlados (Díaz, Coba, Mocha y Mayorga, 2018).

El tercer tópico hace referencia a la inclusión de información de la riqueza hídrica, atmosférica, biótica (flora y fauna) y geológica en la presentación de los estados contables (Mejía y Serna, 2018, p. 109; Florez y Morales, 2019, p. 572), rubros de los estados biocontables frente a los cuales es necesario conocer la aceptación de los expertos que participaron en el estudio. El último aspecto objeto de análisis corresponde a la determinación de la información a revelar en los estados contables, ya sea únicamente financiera o información en función de la sustentabilidad, como lo propone Caro (2011) con respecto a los temas de energía, contaminación, fugas y desperdicios, además de agotamiento, degradación, reducciones y recuperación de afectaciones.

\section{MATERIALES Y MÉTODOS}

La investigación desarrollada en la que se fundamenta este artículo fue cualitativa, descriptiva e inductiva, por cuanto identificó a través de una muestra la postura de los expertos contables en referencia a la pertinencia de la Contabilidad Ambiental para representar la riqueza natural en función de su sustentabilidad; el estudio presenta un carácter explicativo porque se analiza los resultados de los ítems a través de la conceptualización teórica de las escuelas que fundamentan la contabilidad emergente.

Las fuentes de información primaria se obtuvieron mediante encuestas aplicadas a los diferentes grupos de expertos contables a nivel nacional e internacional, a saber, 
grupos de investigadores clasificados por Colciencias-Colombia, semilleros adscritos a los grupos anteriores, gremios profesionales (Colombia), organismos internacionales y directivos académicos de programas de contaduría acreditados de alta calidad al 2016 registrados y visibles en la página del Ministerio de Educación Nacional (MEN) en Colombia.

El estudio tiene una población de 132 expertos contables, directivos académicos, docentes, estudiantes y gremios de instituciones acreditadas en Colombia y organismos internacionales latinoamericanos, de las cuales se seleccionó una muestra representativa que permitió inferir resultados con niveles de confiabilidad altos en relación con la contabilidad convencional y su capacidad para contribuir a la sustentabilidad de la riqueza ambiental.

El cálculo del tamaño de la muestra o la cantidad de expertos contables que suministraron información para sustentar los resultados del estudio se obtuvo a través de la aplicación de la formula N. ${ }^{\circ}$, la cual es usada cuando se conoce el tamaño total de una población.

$$
n=\frac{z^{2} \sigma^{2} N}{z^{2} \sigma^{2}+(N-1) e^{2}} \quad\left(\text { Formula } N .^{o} 1\right)
$$

Con un $\mathrm{z}=-1,96$ valor para un nivel de confianza del $95 \%$ y varianza de 0,25 debido a que no se conoce la varianza, se asume la máxima variabilidad tomando $p=0,5$ y $q=0,5$. El error de muestreo fue $\mathrm{e}=11 \%$; se reemplazaron estos valores en la fórmula $\mathrm{N}^{\circ}{ }^{\circ} 1$, y se obtuvo el total de expertos al cual se le aplicó el instrumento:

$$
n=\frac{1.96^{2} * 0.25 * 132}{196^{2} 0.25+(132-1) * 0.11^{2}}=49.8 \approx 50
$$

El tamaño de la muestra para el presente estudio fue de 50 expertos de un total de 132; para asegurar la representación de expertos de cada grupo se realizó muestreo estratificado, proporcional al total de individuos por grupo. La selección se efectúo de manera independiente en el interior de cada estrato a través de muestreo aleatorio simple. Esto conllevó a que la varianza en cada estrato sea pequeña.

Para el estudio se contó con 5 subgrupos o estratos $(\mathrm{k}=5)$ estratos así:

1. Grupo de investigación

2. Semilleros de investigadores

3. Representantes del gremio profesional

4. Representantes de organismos internacionales
5. Directivos académicos de programas de contaduría acreditados

El tamaño de cada subgrupo es $\mathrm{N}_{1}=43, N_{2}=43, N_{3}=5$, $N_{4}=21, N_{5},=20$ entonces $N=N_{1}+\ldots+N_{5}=132$. Se utilizará la formula $\mathrm{N}^{\circ} 2$ para dividir el tamaño total de la muestra $\mathrm{n}$ en estratos $n_{1}, \ldots, n_{5}$.

$$
n_{i}=n \frac{N_{i}}{N} \quad\left({\text { Formula } \left.N .^{\circ} 2\right)}^{2}\right.
$$

Donde $n=n_{1}+\ldots+n_{5}$

Se conservó la proporción del estrato teniendo en cuenta el número total de expertos en cada uno, de modo que $n_{1}=16, n_{2}=16, n_{3}=2, n_{4}=8, n_{5}=8$ Lo anterior se debe a que el diseño de estratificaciones hace uso de información adicional, pues considera la división de la población de acuerdo con las características relevantes y sirve para reducir el margen de error de muestreo.

\section{RESULTADOS}

La regulación internacional del International Accounting Financial Board (IASB) está reglamentada para la Contabilidad Financiera. El presente estudio pretende identificar la postura de expertos de la comunidad académica contable (tabla 1) con respecto a la pertinencia de los criterios de reconocimiento, medición, presentación y revelación para ser aplicados a la riqueza ambiental en función de su conservación, preservación y sustentabilidad.

La Contabilidad Financiera ha considerado que el beneficio económico es un criterio de reconocimiento de los elementos de los estados contables; el surgimiento de la Contabilidad Ambiental en función de la sustentabilidad natural conlleva a replantear si el beneficio económico es pertinente para determinar si una partida es incluida o excluida de los estados contables.

En la tabla 1 se observa que el $51 \%$ de los expertos considera que este criterio no es pertinente, el 87,5\% de los directivos académicos está de acuerdo con que el beneficio económico no puede ser un criterio de reconocimiento contable para la sustentabilidad de la riqueza ambiental, resultado que permite justificar la búsqueda de otros requisitos para la inclusión de partidas contables ambientales, que incluyan beneficios diferentes a los financieros. Postura contraria tienen los organismos internacionales, pues el $75 \%$ consideran que los beneficios económicos como criterio de reconocimiento son adecuados para la protección de la riqueza ambiental.

El 73\% de los expertos considera válido asociar la medición fiable de los beneficios económicos de una partida como criterio de reconocimiento de la riqueza ambiental 
en función de la sustentabilidad, lineamiento congruente con los paradigmas tradicionales de la Contabilidad. En la tabla 1, se observa que la totalidad de los representantes gremiales expresan que la medición fiable del beneficio económico es pertinente como criterio de reconocimiento; un $87,5 \%$ de los organismos internacionales comparte la misma concepción.

Un análisis comparativo entre los resultados de los beneficios y de la medición evidencian contradicciones por parte de los gremios, pues un 50\% considera que el beneficio económico no es válido como criterio de reconocimiento; sin embargo, el 100\% expresa que la medición fiable del beneficio económico es un criterio que debe tenerse en cuenta.
Comportamiento similar se presenta con los directivos académicos, pues el $87,5 \%$ no comparte que los beneficios económicos sea un criterio de reconocimiento de la riqueza ambiental (tabla 1), a pesar de ello el $62,5 \%$ selecciona la medición monetaria del beneficio como un criterio a tener en cuenta (tabla 2). En síntesis, el 45,5\% de los expertos considera que los criterios tradicionales son pertinentes para la inclusión de partidas en los estados financieros ambientales.

La comunidad académica (estudiantes, profesores y directivos) difiere con los criterios económicos tradicionales como aspectos a tener en cuenta para la Contabilidad Ambiental; en promedio el 55\% de los expertos consultados señaló que

Tabla 1

Apreciación de la comunidad académica con respecto a los aspectos generales de un modelo contable ambiental

\begin{tabular}{|c|c|c|c|c|c|c|c|c|c|c|}
\hline \multirow[t]{2}{*}{ Expertos contables } & \multicolumn{2}{|c|}{$\begin{array}{l}\text { Los beneficios eco- } \\
\text { nómicos asociados } \\
\text { a una partida con- } \\
\text { table es un criterio } \\
\text { pertinente para el } \\
\text { reconocimiento de } \\
\text { la RA en función de } \\
\text { la sustentabilidad }\end{array}$} & \multicolumn{2}{|c|}{$\begin{array}{l}\text { La medición conta- } \\
\text { ble de los beneficios } \\
\text { económicos asocia- } \\
\text { dos a una partida } \\
\text { ambiental es un cri- } \\
\text { terio pertinente para } \\
\text { el reconocimiento de } \\
\text { la riqueza ambiental } \\
\text { en función de su } \\
\text { sustentabilidad }\end{array}$} & \multicolumn{2}{|c|}{$\begin{array}{l}\text { Los métodos de va- } \\
\text { loración de la conta- } \\
\text { bilidad de la conta- } \\
\text { bilidad convencional } \\
\text { son apropiados para } \\
\text { la valoración de la } \\
\text { RA en función de su } \\
\text { sustentabilidad }\end{array}$} & \multicolumn{2}{|c|}{$\begin{array}{l}\text { Los Ítems de infor- } \\
\text { mación a presen- } \\
\text { tar establecidos } \\
\text { en la contabilidad } \\
\text { convencional son } \\
\text { apropiados para la } \\
\text { presentación de los } \\
\text { informes de la R. A }\end{array}$} & \multicolumn{2}{|c|}{$\begin{array}{l}\text { Los ítems de la in- } \\
\text { formación a revelar } \\
\text { establecido en la } \\
\text { contabilidad con- } \\
\text { vencional son apro- } \\
\text { piados para revelar } \\
\text { la información de la } \\
\text { R.A en función de la } \\
\text { sustentabilidad }\end{array}$} \\
\hline & No & Si & No & Si & No & Si & No & Si & No & Si \\
\hline Directivo académico & $87,5 \%$ & $12,5 \%$ & $37,5 \%$ & $62,5 \%$ & $100,0 \%$ & $0,0 \%$ & $100,0 \%$ & $0,0 \%$ & $87,5 \%$ & $12,5 \%$ \\
\hline Docente & $50,0 \%$ & $50,0 \%$ & $43,8 \%$ & $56,8 \%$ & $75,0 \%$ & $25,0 \%$ & $75,0 \%$ & $25,0 \%$ & $75,0 \%$ & $25,0 \%$ \\
\hline Estudiantes & $43,8 \%$ & $56,8 \%$ & $43,8 \%$ & $56,8 \%$ & $87,5 \%$ & $12,5 \%$ & $50,0 \%$ & $50,0 \%$ & $75,0 \%$ & $25,0 \%$ \\
\hline Gremio & $50,0 \%$ & $50,0 \%$ & $0,0 \%$ & $100,0 \%$ & $100,0 \%$ & $0,0 \%$ & $100,0 \%$ & $0,0 \%$ & $100,0 \%$ & $0,0 \%$ \\
\hline Organismo Internacional & $25,0 \%$ & $75,0 \%$ & $12,5 \%$ & $87,5 \%$ & $75,0 \%$ & $25,0 \%$ & $75,0 \%$ & $25,0 \%$ & $62,5 \%$ & $37,5 \%$ \\
\hline Promedio & $51 \%$ & $49 \%$ & $28 \%$ & $73 \%$ & $87,5 \%$ & $12,5 \%$ & $80 \%$ & $20 \%$ & $80 \%$ & $20 \%$ \\
\hline
\end{tabular}

Fuente: construcción propia con base en encuestas a expertos contables.

Tabla 2

Criterios de reconocimiento de los elementos de los estados contables ambientales

\begin{tabular}{|c|c|c|c|c|c|c|c|c|}
\hline \multirow[t]{2}{*}{ Expertos contables } & \multicolumn{2}{|c|}{$\begin{array}{c}\text { Beneficios económicos } \\
\text { de la R. A }\end{array}$} & \multicolumn{2}{|c|}{$\begin{array}{l}\text { Medición confiable de } \\
\text { los beneficios } \\
\text { económicos de la R. A }\end{array}$} & \multicolumn{2}{|c|}{ Existencia de la R. A } & \multicolumn{2}{|c|}{$\begin{array}{c}\text { Control de las } \\
\text { organizaciones sobre } \\
\text { la } \mathrm{R} . \mathrm{A}\end{array}$} \\
\hline & No & Sí & No & Sí & No & Sí & No & Sí \\
\hline Directivo académico & $100,0 \%$ & $0,0 \%$ & $62,5 \%$ & $37,5 \%$ & $62,5 \%$ & $37,5 \%$ & $0 \%$ & $100 \%$ \\
\hline Docente & $53,8 \%$ & $46,2 \%$ & $62,5 \%$ & $37,5 \%$ & $31,3 \%$ & $68,7 \%$ & $25 \%$ & $75 \%$ \\
\hline Estudiantes & $81,3 \%$ & $18,7 \%$ & $62,5 \%$ & $37,5 \%$ & $31,3 \%$ & $68,7 \%$ & $31,3 \%$ & $69 \%$ \\
\hline Gremio & $50,0 \%$ & $50,0 \%$ & $0,0 \%$ & $100,0 \%$ & $100,0 \%$ & $0,0 \%$ & $100 \%$ & $0 \%$ \\
\hline Organismo Internacional & $50,0 \%$ & $50,0 \%$ & $25,0 \%$ & $75,0 \%$ & $50,0 \%$ & $50,0 \%$ & $50 \%$ & $50 \%$ \\
\hline Promedio & $67 \%$ & $33 \%$ & $43 \%$ & $58 \%$ & $55 \%$ & $45 \%$ & $41 \%$ & $59 \%$ \\
\hline
\end{tabular}

Fuente: construcción propia con base en encuestas a expertos contables. 
la inclusión de la riqueza ambiental en los estados contables no debe estar sujeta al cumplimiento de los criterios económicos tradicionales (tabla 2).

Criterios de reconocimiento emergentes de la riqueza ambiental (Biocontabilidad)

La Biocontabilidad propone dos criterios de reconocimiento de los elementos de los estados biocontables, de la existencia de la riqueza ambiental y del control organizacional de esta.

El porcentaje promedio de aceptación de nuevos criterios de reconocimiento para una contabilidad emergente según los resultados de la tabla 2 es del $52 \%$, puntaje que no muestra una tendencia significativamente alta para su implementación futura. A pesar de la evidente crisis ambiental que se observa a través del agotamiento y degradación de los ecosistemas, los resultados reflejan la fuerte aceptación que tiene la Contabilidad Económica (tradicional), la cual ha contribuido a fortalecer la acumulación de capital financiero, en gran medida soportada en el deterioro del patrimonio natural.

La postura anterior evidencia la aceptación de la comunidad contable del paradigma clásico amparado en el consumo y en la utilidad económica, lo que ha impedido la apertura a nuevos modelos que conduzcan a la modificación o ampliación del instrumental contable que permita una rendición de cuentas tendiente a la protección, conservación y preservación dinámica de la riqueza ambiental. Esta situación justifica la necesidad de ampliar el trabajo de sensibilización sobre la importancia y el valor de los recursos naturales y de la responsabilidad de la contabilidad para contribuir a una sustentabilidad integral.

El instrumento aplicado permitió conocer el porcentaje de aceptación de los criterios tanto tradicionales como emergentes para el reconocimiento contable; de la tabla 2 se infiere que en promedio el $48,7 \%$ de los expertos consultados seleccionaron los cuatro criterios para el reconocimiento de la riqueza ambiental. Los resultados obtenidos ratifican la necesidad de desarrollar criterios específicos para partidas ambientales, los cuales se obtendrán de investigaciones de carácter interdisciplinario, donde la participación de expertos en ciencias de la vida y la tierra serán determinantes para potenciar el saber contable al servicio de la sustentabilidad.

Algunos expertos encuestados consideraron que se debe tener en cuenta para la valoración de la riqueza ambiental en función de la sustentabilidad los siguientes criterios de reconocimiento: los impactos y la proyección de la R. A., los beneficios ambientales, los intercambios y la capacidad de los ecosistemas para proveer bienes y servicios ambientales, la resiliencia, los balances de masa, hídricos y energéticos que incluyen los de transferencia de información.

Métodos de valoración tradicional y emergente para representar la riqueza ambiental

La Contabilidad Financiera tradicional utiliza para la representación de los elementos de los estados contables el método cuantitativo monetario, aplicando como bases de medición el costo histórico, el valor corriente, el valor razonable, el valor en uso y valor de cumplimiento y costos corrientes (International Accounting Financial Board, 2018, p. 51).

La expresión monetaria no incluye el valor total de los recursos ecosistémicos, tal medición no logra representar los beneficios sociales y ambientales que estos generan. La información organizacional lograría tener un carácter integral a través de la inclusión de partidas no financieras de estos recursos, permitiendo conocer en detalle los efectos de los procesos económico-empresariales en la naturaleza y en la sociedad.

El estudio evidencia la necesidad de formular nuevos métodos de valoración que permitan la inclusión de otras manifestaciones de la riqueza no representables por los métodos financieros; esta postura es congruente con la apreciación expresada por el $87,5 \%$ de la comunidad académica (tabla 1), lo que refleja un desacuerdo con la valoración tradicional como única alternativa utilizada en los procesos contables.

Se evidencia que los directivos académicos y gremios consideran por unanimidad que los métodos de valoración contable convencional no son apropiados para la sustentabilidad de la riqueza ambiental.

Dentro de los métodos alternativos propuestos para la valoración de la riqueza ambiental, el método multicriterio obtuvo un porcentaje del $76 \%$ de aceptación (tabla 3), el cual obtuvo la mayor aprobación por parte de los expertos encuestados; el 89\% no estuvo de acuerdo en incluir solo el método cuantitativo monetario para la representación de la riqueza ambiental.

La Contabilidad tradicional en sus estados financieros presenta únicamente rubros de carácter económico que son representados y medibles de manera confiable en términos monetarios, tales como inventarios, propiedad planta y equipo, instrumentos financieros, activos intangibles, propiedades de inversión, activos biológicos y pasivos, entre otros; partidas que en promedio el $80 \%$ (tabla 1) de los expertos encuestados considera no son apropiadas para la presentación de la riqueza ambiental en función de su sustentabilidad. 
El 100\% de los directivos y gremios (tabla1) considera que los rubros de presentación tradicionales no son los adecuados para expresar en los estados contables la totalidad de aspectos relacionados con la riqueza ambiental, por lo cual es necesario indagar sobre otros tópicos que permitan mediciones integrales para su sustentabilidad (Vaca y Ramírez, 2018). Los resultados justifican el desarrollo de la Biocontabilidad como campo emergente, la cual propone nuevos conceptos y cuentas para la presentación de informes de la riqueza ambiental.

Información a presentar en la contabilidad tradicional $y$ emergente para representar la riqueza ambiental

La Biocontabilidad como disciplina tiene como finalidad contribuir a la óptima acumulación, generación, distribución y sustentabilidad de la riqueza ambiental, para lo cual propone la inclusión de nuevos rubros no contemplados en la Contabilidad tradicional; postura que es respaldada por expertos de este campo del saber, que en promedio el 79,5\% (tabla 4) está de acuerdo en incluir nuevas cuentas, tales como la hídrica, la atmosférica, la biótica y la geológica.

Las categorías presentadas como posibles cuentas en la tabla 4 pueden ser desagregadas en niveles que permitan una mejor comprensión de la existencia y circulación de los recursos naturales; por ejemplo, en el recurso hídrico se puede detallar aspectos como las aguas superficiales, marinas, subterráneas, nieve y hielo; además de información sobre los aspectos de calidad, temperatura y recarga; en lo atmosférico se debe tener en cuenta aspectos de calidad, clima y temperatura; en el recurso de la flora se debe clasificar por árboles, arbustos, hierbas, cosechas, microflora, plantas acuáticas, especies en peligro y corredores; así entre otros recursos (Franco, 2015).

Algunos de los expertos proponen como tópicos de presentación de la información contable ambiental a la riqueza energética y a la conservación social (considerando

Tabla 3

Métodos de valoración de la riqueza ambiental

\begin{tabular}{|c|c|c|c|c|c|c|c|c|}
\hline \multirow[t]{2}{*}{ Expertos contables } & \multicolumn{2}{|c|}{ Métodos cualitativos } & \multicolumn{2}{|c|}{$\begin{array}{l}\text { Métodos cuantitativos } \\
\text { monetarios }\end{array}$} & \multicolumn{2}{|c|}{$\begin{array}{c}\text { Métodos cuantitativos } \\
\text { no monetarios }\end{array}$} & \multicolumn{2}{|c|}{ Métodos multicriterio } \\
\hline & No & $\mathrm{Si}$ & No & $\mathrm{Si}$ & No & Si & No & $\mathrm{Si}$ \\
\hline Directivo Académico & $87,5 \%$ & $100,0 \%$ & $100,0 \%$ & $0,0 \%$ & $62,5 \%$ & $37,5 \%$ & $25,0 \%$ & $75,0 \%$ \\
\hline Docente & $37,5 \%$ & $62,5 \%$ & $68,8 \%$ & $31,2 \%$ & $31,3 \%$ & $68,7 \%$ & $31,3 \%$ & $68,7 \%$ \\
\hline Estudiantes & $43,8 \%$ & $56,2 \%$ & $100,0 \%$ & $0,0 \%$ & $31,3 \%$ & $68,7 \%$ & $50,0 \%$ & $50,0 \%$ \\
\hline Gremio & $100,0 \%$ & $0,0 \%$ & $100,0 \%$ & $0,0 \%$ & $100,0 \%$ & $0,0 \%$ & $0,0 \%$ & $100,0 \%$ \\
\hline Organismo Internacional & $75,0 \%$ & $25,0 \%$ & $75,0 \%$ & $25,0 \%$ & $75,0 \%$ & $25,0 \%$ & $12,5 \%$ & $87,5 \%$ \\
\hline Promedio & $69 \%$ & $49 \%$ & $89 \%$ & $11 \%$ & $60 \%$ & $40 \%$ & $24 \%$ & $76 \%$ \\
\hline
\end{tabular}

Fuente: construcción propia con base en encuestas a expertos contables.

Tabla 4

Cuentas emergentes para la presentación de información de la riqueza ambiental

\begin{tabular}{|c|c|c|c|c|c|c|c|c|}
\hline \multirow[t]{2}{*}{ Expertos contables } & \multicolumn{2}{|c|}{ Riqueza hídrica } & \multicolumn{2}{|c|}{ Riqueza Atmosférica } & \multicolumn{2}{|c|}{$\begin{array}{c}\text { Riqueza bioética (flora } \\
\text { y fauna) }\end{array}$} & \multicolumn{2}{|c|}{ Riqueza geológica } \\
\hline & No & Sí & No & Sí & No & Sí & No & Sí \\
\hline Directivo académico & $25,0 \%$ & $75,0 \%$ & $50,0 \%$ & $50,0 \%$ & $25,0 \%$ & $75,0 \%$ & $37,5 \%$ & $62,5 \%$ \\
\hline Docente & $0,0 \%$ & $100,0 \%$ & $12,5 \%$ & $87,5 \%$ & $0,63 \%$ & $99,4 \%$ & $12,5 \%$ & $87,5 \%$ \\
\hline Estudiantes & $18,8 \%$ & $81,2 \%$ & $43,8 \%$ & $56,2 \%$ & $25,0 \%$ & $75,0 \%$ & $50,0 \%$ & $50,0 \%$ \\
\hline Gremio & $0,0 \%$ & $100,0 \%$ & $0,0 \%$ & $100,0 \%$ & $0,0 \%$ & $100,0 \%$ & $0,0 \%$ & $100,0 \%$ \\
\hline Organismo Internacional & $12,5 \%$ & $87,5 \%$ & $50,0 \%$ & $50,0 \%$ & $12,5 \%$ & $87,5 \%$ & $37,5 \%$ & $62,5 \%$ \\
\hline Promedio & $11 \%$ & $89 \%$ & $31 \%$ & $69 \%$ & $13 \%$ & $87 \%$ & $28 \%$ & $73 \%$ \\
\hline
\end{tabular}

Fuente: construcción propia con base a encuesta a expertos contables 
la acción antrópica en la misma); además, a los flujos de información de la contabilidad de la naturaleza hacia la contabilidad económica y social.

La Contabilidad tradicional ha revelado información asociada a detalles y explicaciones relacionadas con las partidas monetarias, las cuales solo dan respuesta a la existencia y circulación de recursos económicos presentados en los estados financieros. Los ítems anteriores no son apropiados para la revelación de información de la riqueza ambiental, postura apoyada por el $80 \%$ de los encuestados como se puede evidenciar en la tabla 1.

La no aceptación de los ítems de la Contabilidad tradicional para la revelación de información contable de la rique$\mathrm{za}$ ambiental es respaldada por el $100 \%$ de los expertos del sector de los gremios, posición que coincide con los postulados de la Biocontabilidad, la cual considera que los informes contables deben revelar información cuantitativa no monetaria y cualitativa de carácter diverso.

Información por revelar en la contabilidad emergente para representar la riqueza ambiental

La Biocontabilidad propone unos campos de revelación de información como se presenta en la tabla 5, los cuales son aceptados por el 54,03\% de los encuestados para ser incluidos en los informes contables de la riqueza ambiental. La Contabilidad debe predecir el comportamiento futuro de la riqueza, de manera que permita una adecuada y oportuna toma de decisiones por parte de los usuarios para contribuir a la protección, conservación y sustentabilidad de la riqueza ambiental; además, es importante la inclusión de políticas y actividades tendientes a la preservación dinámica de la naturaleza, estas intervenciones se denominan prescripciones, las cuales deben estar soportadas en rigurosas predicciones.

El 51\% y $49 \%$ de los expertos consideran, respectivamente, que la predicción y la prescripción son aspectos que no se deben revelar en los informes contables; postura que evidencia el reduccionismo que se tiene frente a este saber. Contraria a la visión anterior, la Biocontabilidad considera que se requieren predicciones que permitan la intervención anticipada de acciones humanas, con el objetivo de alcanzar situaciones deseables de conformidad con el interés general y la defensa de la vida digna en todas sus formas.

Los resultados de la tabla 5 justifican el desarrollo de actividades en la comunidad científica, académica e investigativa de la Biocontabilidad que impulsen el fortalecimiento de los componentes teóricos y conceptuales de este campo disciplinario, a través de la inclusión de nuevas visiones críticas que permitan ampliar su interpretación y comprensión, de forma que al ser considerado un saber científico, los objetivos de predicción y prescripción sean determinantes en los procesos de preparación y presentación de estados e informes contables.

Formulación de una nueva disciplina contable para la riqueza ambiental

La formulación de una nueva disciplina contable que estudie la riqueza ambiental con el propósito de contribuir a su preservación es aceptada por el $76 \%$ de los expertos encuestados, como se puede observar en la tabla 6 , en la cual los estudiantes y los organismos profesionales otorgan una mayor ponderación a estos cambios para la ciencia contable.

La justificación expresada por los expertos que respondieron positivamente a la formulación de una nueva disciplina contable de la riqueza ambiental se sintetiza en los siguientes términos: la Contabilidad, en sus orígenes, fue de carácter no monetario, el proceso de monetización es posterior.

En tal sentido, una nueva disciplina implica recuperar los campos de actuación original del saber contable,

Tabla 5

Campos emergentes para la revelación de información de la riqueza ambiental

\begin{tabular}{|c|c|c|c|c|c|c|c|c|}
\hline \multirow[t]{2}{*}{ Expertos contables } & \multicolumn{2}{|c|}{ Descripción } & \multicolumn{2}{|c|}{ Explicación } & \multicolumn{2}{|c|}{ Predicción } & \multicolumn{2}{|c|}{ Prescripción } \\
\hline & No & Sí & No & Sí & No & Sí & No & Sí \\
\hline Directivo académico & $25,0 \%$ & $75,0 \%$ & $62,5 \%$ & $37,5 \%$ & $25,0 \%$ & $75,0 \%$ & $75,0 \%$ & $25,0 \%$ \\
\hline Docente & $12,5 \%$ & $87,5 \%$ & $25,0 \%$ & $75,0 \%$ & $56,3 \%$ & $43,7 \%$ & $56,3 \%$ & $43,7 \%$ \\
\hline Estudiantes & $37,5 \%$ & $62,5 \%$ & $25,0 \%$ & $75,0 \%$ & $75,0 \%$ & $25,0 \%$ & $68,8 \%$ & $31,2 \%$ \\
\hline Gremio & $0,0 \%$ & $100,0 \%$ & $50,0 \%$ & $50,0 \%$ & $50,0 \%$ & $50,0 \%$ & $100,0 \%$ & $0,0 \%$ \\
\hline Organismo Internacional & $50,0 \%$ & $50,0 \%$ & $12,5 \%$ & $87,5 \%$ & $50,0 \%$ & $50,0 \%$ & $62,5 \%$ & $37,5 \%$ \\
\hline Promedio & $25 \%$ & $75 \%$ & $35 \%$ & $65 \%$ & $51 \%$ & $49 \%$ & $73 \%$ & $27 \%$ \\
\hline
\end{tabular}

Fuente: construcción propia con base a encuesta a expertos contables 
Tabla 6

Aceptación de la formulación de nueva disciplina contable para contabilizar la riqueza ambiental en función de su sustentabilidad

\begin{tabular}{lcc}
\hline $\begin{array}{l}\text { Expertos contables } \\
\text { No requiere la formulación de una nueva disciplina contable (diferente a la convencional) para contabilizar } \\
\text { la riqueza ambiental en función de su sustentabilidad }\end{array}$ & $\begin{array}{c}\text { Se } \\
\text { Sí }\end{array}$ \\
\hline Directivo académico & $25,0 \%$ & $75,0 \%$ \\
Docente & $31,3 \%$ & $68,7 \%$ \\
Estudiantes & $0,63 \%$ & $\mathbf{9 9 , 4 \%}$ \\
Gremio & $50,0 \%$ & $\mathbf{5 0 , 0} \%$ \\
Organismo Internacional & $12,5 \%$ & $\mathbf{8 7 , 5 \%}$ \\
Promedio & $\mathbf{2 4 \%}$ & $\mathbf{7 6 \%}$ \\
\hline
\end{tabular}

Fuente: construcción propia con base en encuestas a expertos contables

donde se incluya información con el propósito de satisfacer las necesidades de usuarios diferentes a los establecidos en la regulación contable tradicional, logrando superar las deficiencias y limitaciones del enfoque financiero y contribuir en el control de riesgos futuros de carácter ambiental a través de la implementación de métodos predictivos que permitan la adopción de medidas de protección y salvaguarda de los equilibrios naturales. Por ende, es necesario redefinir y resignificar el lenguaje y la práctica contable, es posible que los conceptos de riqueza, capital, patrimonio, ingreso, entre otros, no sean pertinentes para una nueva comprensión de la contabilidad en función de la sustentabilidad.

Los expertos que no están de acuerdo con la formulación de una nueva disciplina contable de carácter ambiental consideran que la Contabilidad tradicional se puede reestructurar para la representación de la riqueza natural. Para ello se requiere adaptar los conceptos y procedimientos tradicionales, a través de la participación de profesionales de otras disciplinas y del aporte de estudios científicos relacionados con los temas ambientales. Postura contraria a la expuesta por la Biocontabilidad, que considera que la corriente tradicional del saber contable es reduccionista en los aspectos ontológicos, epistemológicos, axiológicos y técnicos; situación que exige cambios fundamentales en su estructura teórica, conceptual y procedimental.

\section{DISCUSIÓN}

La propuesta de diseñar un sistema contable (Biocontabilidad) en función de la sustentabilidad natural, replantea el beneficio económico como criterio pertinente para determinar si una partida es incluida o excluida de los estados contables (Ceballos y Mejía, 2016 y Álvarez, 2019). La investigación reveló que el 50\% de encuestados considera la necesidad de ampliar dichos criterios tradicionales. Un porcentaje significativo de expertos está de acuerdo con la necesidad de ampliar los criterios de valoración para la representación de la riqueza ambiental, lo cual permite que, además de la utilización de unidades monetarias tradicionales, la riqueza ambiental pueda ser expresada a través de mediciones de carácter biogeofísico que promuevan otros marcos de análisis donde se tengan en cuenta las interacciones y dinámicas propias de la naturaleza, posición que se encuentra en la línea de la Teoría Tridimensional de la Contabilidad T3C. La postura de los expertos muestra que la orientación de los Estándares Internacionales de Reportes Financieros (IFRS) no puede ser el único criterio de reconocimiento; las propuestas de la Economía Ecológica, la Bioeconomía, las ciencias de la tierra y de la vida deben ser consideradas. La mayoría de los expertos considera que las descripciones, explicaciones, predicciones y prescripciones asociadas a los rubros monetarios no son apropiadas para revelar procesos relacionados con la riqueza ambiental en función de su sustentabilidad. La Biocontabilidad propone superar el enfoque monetario de la contabilidad tradicional con la promoción de la revelación de información cuantitativa no monetaria y cualitativa de carácter diverso, que permita salvaguardar los recursos naturales y sus relaciones ecosistémicas. Contraria a la visión de los científicos de otros campos del saber donde la predicción y la prescripción son etapas fundamentales de la ciencia, en Contabilidad, los expertos consideran que estos aspectos no deben ser objeto de revelación, postura que evidencia el reduccionismo teórico y práctico en la visión de la disciplina. La regulación contable internacional tanto del sector privado (IASB) como del público (IPSASB) está limitada a la presentación de estados financieros que reflejan la riqueza pasada y presente de los activos, pasivos y patrimonios, y sus movimientos a través de los ingresos y egresos. Los expertos contables consideran la 
importancia de avanzar en las predicciones y prescripciones, situaciones asociada a la gestión organizacional y la futura situación y flujos de la riqueza ambiental, social y económica. El análisis general del estudio realizado permite concluir que, la mayoría de integrantes de la comunidad académica contable está de acuerdo con la formulación de una nueva disciplina (diferente a la convencional) para contabilizar la riqueza ambiental en función de su sustentabilidad que viabilice la inclusión de los recursos naturales y sus relaciones en los diferentes reportes organizacionales financieros y no financieros.

\section{REFERENCIAS}

Álvarez, A. (2006). Gestión contable bajo USGAAP. México: Alfaomega.

Álvarez, H. (2019). Buscando la ruta de la medición-valoración ecológica no monetaria, en la Teoría Tridimensional de la Contabilidad. Revista Visión Contable, (20), 22-95. doi:10.24142/rvc.n20a2

Ceballos, O. y Mejía, E. (2016). Medición contable de la sustentabilidad organizacional [método de circulación]. Un enfoque desde la Teoría Tridimensional de la Contabilidad. Revista Libre Empresa, (25),127-142. ISSN: 1657-2815.

Caro, J. (2011). Contabilidad ambiental. Ventajas del desarrollo sustentable. Buenos Aires: EDICON.

Cooper, W. e Ijiri, Y. (2005). Diccionario Kohler para contadores. México: Limusa.

Diaz, J.; Coba, E.; Mocha, J. y Mayorga, M. J. (2018). La Biocontabilidad en la conservación de los recursos turísticos naturales: caso de estudio de playa de Salinas, Ecuador. Revista Espacios, 39(16), 31-44.

Fowler, E. (2008). Diccionario de contabilidad y auditoría. Buenos Aires: La Ley.

Franco, J. (2015). Evaluación del impacto ambiental. México: Trillas.
Florez, Á. y Morales, M. (2019). Gestión contable ambiental en empresas manufactureras generadores de residuos peligros. Revista científica General José María Córdova, 17(25), 557-578. doi.org/10.21830/19006586.383

García, C.; Rodríguez, D. y Ruiz, J. (2016). La heterogeneización de magnitudes en la teoría tridimensional de la contabilidad. Libre Empresa, 13(1), 161-176. doi. org/10.18041/libemp.2016.v13n1.25107

Gómez-Baggethun, E. y De Groot, R. (2007). Capital natural y funciones de losecosistemas: explorando las bases ecológicas de la economía. Ecosistemas, 16(3), 4-14.

Grajales, G. y Sánchez, W. (2004). Contabilidad conceptual e instrumental. Pereira: Investigar Editores.

International Accounting Financial Board. (2016). Normas NIIF. Londres: IFRSF.

International Accounting Financial Board. (2018). Conceptual Framework for Financial Reporting. Londres: IFRSF.

International Public Sector Accounting Standards Board. (2014). Marco Conceptual para la información financiera con propósito general de las entidades del sector público. New York: IFAC.

Mejía, E. y Ceballos, O. (2016). Medición contable de la sustentabilidad organizacional desde la Teoría Tridimensional de la Contabilidad. Revista General José María Córdova, 14(18), 215-243. ISSN: 1900-6586.

Mejía, E. y Serna, C. (2018). Tránsito de la contabilidad convencional a la biocontabilidad. Cali: REDICEAC.

Suárez, J.; Cárdenas, M.; Méndez, V.; Rojas, L.; Castillo, I.; Wilches, C.; Contento, L. y Varela, J. (2020). Cuentas y cuentos... cuidad de las cuentas públicas: custodia de la probidad. Bogotá: FUSM-CGN.

Vaca, A. y Ramírez, D. (2018). Contabilidad de la cultura para el desarrollo sostenible. Espacios, 39 (44), 13-25. 
